\title{
Estudio sobre la satisfacción laboral y la felicidad en médicos dermatólogos
}

\author{
Leonel Fierro-Arias ${ }^{1}$, Pilar Simón-Díaz', Rosa María Ponce-Olivera y Roberto Arenas-Guzmán² \\ ${ }^{1}$ Servicio de Dermatología, Hospital General de México Dr. Eduardo Liceaga; ${ }^{2}$ Servicio de Micología, Hospital General Dr. Manuel Gea González. \\ Ciudad de México, México
}

\begin{abstract}
Resumen
Objetivo: Conocer la felicidad y la satisfacción laboral de los dermatólogos en México. Método: Estudio descriptivo, mediante encuesta en línea dirigida a dermatólogos especialistas, graduados y con ejercicio en México. Se incluyeron elementos demográficos, el Índice de Felicidad de Pemberton (con validación local) y preguntas para evaluar la satisfacción personal. Se realizaron medidas de tendencia central y dispersión; para comparar las variables categóricas, se utilizaron tablas de contingencia para ji al cuadrado, y al comparar variables cuantitativas con distribución normal se utilizó la prueba t de Student. Resultados: Se incluyeron 219 encuestados, el $72.6 \%$ mujeres y el 27.4\% hombres, con una media de edad de 45.6 años y un promedio de 16 años de ejercicio profesional. La mayoría (64.8\%) eran egresados de la Ciudad de México y el $75.3 \%$ estaban muy satisfechos con su escuela de formación. Un 93\% se manifestó muy satisfecho con su especialidad y el $98.6 \%$ volvería a escogerla; el mayor motivo sería abarcar ámbitos médicos y quirúrgicos. El grado de felicidad según el Índice de Pemberton fue «alto» (media: 9.11; desviación estándar: 1.73). Conclusión: Este primer estudio en América Latina sobre este aspecto en dermatólogos, muestra unos altos índices de satisfacción y felicidad, tanto en el área profesional como en la personal.
\end{abstract}

PALABRAS CLAVE: Dermatología. Satisfacción. Felicidad. Médicos.

\begin{abstract}
Objective: To assess the level of happiness and satisfaction in the life and medical practice of dermatologists in Mexico. Method: A descriptive study (online survey) was conducted focused on practicing dermatologists in our country. Questions included demographic characteristics, the Pemberton happiness index (with local validation) and questions that assessed the degree of personal satisfaction. Descriptive statistics were used to obtain the central tendency and dispersion. Measures of central tendency and dispersion were performed; to compare categorical variables, contingency tables for chi-square test were used and when comparing quantitative variables with normal distribution, Student's $t$ t-test was used. Results: 219 surveys were included, $72.6 \%$ female and $27.4 \%$ male, with an average age of 45.6 and an average of 16 years of medical practice. Most of them (64.8\%) graduate from Mexico City; $93 \%$ were very satisfied with the specialty and $98.6 \%$ of them would choose the same once again, the most important reason is to encompass medical and surgical areas. The level of happiness by using the Pemberton scale was "high" (mode: 9.11; standard deviation: 1.73). Conclusions: This first study in Latin America on this subject in dermatologists showed high levels of satisfaction and happiness in both professional and personal areas.
\end{abstract}

KEY WORDS: Dermatology. Satisfaction. Happiness. Doctors.

\author{
Correspondencia: \\ Leonel Fierro-Arias \\ Perpetua 40-3 piso \\ Col. San José Insurgentes, Benito Juárez \\ C.P. 03900 , Ciudad de México, México \\ Email: leofierro@yahoo.com
}

Fecha de recepción en versión modificada: 16-07-2016 Fecha de aceptación: 18-08-2016 DOI://dx.doi.org/10.24875/GMM.17002763
Gac Med Mex. 2018;154:26-35

Contents available at PubMed www.gacetamedicademexico.com 


\section{Introducción}

En años recientes y en varias regiones del mundo se ha incrementado la atención a los asuntos relacionados con la satisfacción laboral entre los profesionales de la salud. Este concepto se relaciona directamente con el bienestar de los médicos y con la calidad del servicio que, en consecuencia, proveen a sus pacientes. Los estudios científicos de satisfacción o bienestar son relativamente recientes; sin embargo, la importancia que los individuos hemos dado a sentirnos bien o a albergar percepciones de felicidad tiene su origen miles de años atrás y ha conformado algunas de las tradiciones del pensamiento más importantes de la humanidad'.

Según la Organización Mundial de la Salud, la salud mental es «un estado de bienestar en el cual uno es consciente de sus propias capacidades, puede afrontar las tensiones normales de la vida y trabajar de forma productiva, y además es capaz de contribuir a la comunidad»?2.

El bienestar tiene dos componentes: el hedónico, que consiste en el placer o bienestar momentáneo, y el eudaimónico, que se enfoca en la autorrealización, el crecimiento psicológico, las metas y las necesidades a mediano y largo plazo ${ }^{3}$. Una propuesta de modelo multidimensional del bienestar psicológico eudaimónico integra los siguientes aspectos: la autoaceptación (sensación de sentirse bien consigo mismo), las relaciones positivas con los demás, el control del ambiente en donde se desenvuelve, la autonomía, el tener un propósito en la vida y el crecimiento personal ${ }^{4}$. Precisamente por este papel central en el funcionamiento humano, la evaluación del bienestar es una tarea de gran importancia y, a la vez, un interesante reto científico ${ }^{5}$.

La satisfacción en el trabajo depende tanto de las condiciones ambientales como de las características del entorno y de los procesos mentales del individuo. Los componentes del entorno laboral son diversos y algunos de ellos no se logran modificar, pero sin duda resultan relevantes, pues causan impacto en la percepción del individuo, sin olvidar que los elementos de felicidad o infelicidad también se originan en las personas.

En esta concepción, existen dos aspectos importantes: el primero son los procesos que pueden concretarse a corto plazo (la forma de afrontar y pensar en situaciones específicas), cuando la felicidad se encuentra determinada parcialmente por los juicios comparativos, y el segundo se establece por la evaluación relacionada con la autoeficacia, la novedad y la relevancia personal. Así, una de las características relevantes de la satisfacción laboral es que la persona perciba una autorrealización (grado en que se valoran la autonomía, los nuevos aprendizajes, etc.) ${ }^{6}$.

Otros elementos se establecen a largo plazo (aspectos de disposición o demográficos), entre los que se encuentran los rasgos de personalidad y el género. De este modo, las variables ambientales e individuales interactúan de manera particular en cada persona. Por otra parte, las características cognitivas, físicas o de temperamento influyen en la elección de profesiones y empleos, o en la inclinación por determinadas actividades en un trabajo, lo que a su vez incide en la felicidad ${ }^{6}$.

Encuestas en distintos países muestran que los dermatólogos tienen mayor satisfacción laboral y menor desgaste profesional que otros médicos. Hasta donde tenemos conocimiento, y tras una búsqueda de información, no existen en nuestro país, ni en el resto de América Latina, evaluaciones sobre los grados de satisfacción y felicidad en los practicantes de esta especialidad.

Los objetivos del presente estudio fueron evaluar la satisfacción y el bienestar de los dermatólogos en México, conocer si esta rama de la medicina ha cumplido sus expectativas profesionales, comparar el grado de satisfacción y felicidad de la población estudiada con respecto a las investigaciones reportadas en otros países, y asociar el grado de satisfacción con la escuela donde realizaron sus estudios en dermatología, así como con algunas características demográficas.

\section{Métodos}

Se realizó un estudio descriptivo, tipo encuesta en línea, que contenía elementos para obtener datos demográficos, el Índice de Felicidad de Pemberton (IFP, The Pemberton Happiness Index) y algunas preguntas que evaluaron el grado de satisfacción con la profesión y la escuela de formación de posgrado.

Tras un consentimiento informado y con reservas de confidencialidad de datos personales, se encuestó a médicos dermatólogos que ejercen en nuestro país, que fueran egresados de alguna escuela mexicana con formación de especialistas y que aceptaran participar en el estudio, independientemente de su edad, sexo, años de ejercicio y ámbito de trabajo (institucional, privado o ambos). Para el cálculo del tamaño de muestra se empleó la ecuación para poblaciones 
infinitas sin reemplazamiento, con base en una proporción. En este caso, el resultado fue 0.7, con un nivel de confianza del $95 \%$ y un margen de error del $6 \%$; el tamaño de muestra arrojó una $n=224$.

La base de datos se obtuvo con información otorgada por las principales agrupaciones nacionales de la especialidad. Por correo electrónico, a todos los sujetos de estudio se les envió una carta de invitación, la encuesta con los elementos descritos y el IFP, utilizando la herramienta electrónica Google Formats ${ }^{\odot}$. Después se evaluó el nivel de satisfacción con la profesión y la escuela donde estudiaron, mediante instrumentos estandarizados.

Se realizó análisis descriptivo para las variables edad (años), ejercicio profesional (años), satisfacción con la vida profesional, energía para cumplir las tareas cotidianas, sentir que la vida profesional es útil y valiosa, y sentirse satisfecho con su forma de ser. Se obtuvieron la media, la desviación estándar o típica, los valores máximo y mínimo, y los percentiles 25, 50 y 75 .

Con las variables sexo, escuela de formación, satisfacción con la especialidad y con la escuela de formación, motivo más relevante de satisfacción y volvería a estudiar dermatología se obtuvieron sus frecuencias expresadas en porcentajes, así como gráficas de barras y sectores para las variables cualitativas, e histogramas para las variables cuantitativas.

El valor del IFP en cada individuo se obtuvo mediante tres pasos: 1) el promedio de 10 variables que integran el «bienestar recordado» (escala eudaimónica), 2) el promedio de 10 variables que forman el «bienestar experimentado» (escala hedónica), y 3) la suma de estos promedios. La interpretación de los valores resultantes es la siguiente: de 0 a 3.70 , muy bajo; de 3.71 a 5.90 , bajo; de 5.91 a 7.90 , medio; de 7.91 a 9.2, alto; y de 9.2 a 10, muy alto.

Para el procesamiento de la información se elaboró una base de datos en Excel de Microsoft ${ }^{\circledR}$, y el análisis estadístico se realizó con el paquete computacional Statistical Package for the Social Sciences ${ }^{\circledR}$ (SPSS) versión 22.

EI IFP se obtiene por una escala integral validada en siete diferentes idiomas, culturas y países, incluyendo al nuestro ${ }^{8}$. Contiene 10 elementos relacionados con los diferentes dominios de bienestar recordado (general, eudaimónico, hedónico), además de 11 elementos de bienestar experimentado, que pueden ser transformados a un índice único de bienestar.

La primera dimensión de análisis de la escala fue la «dimensionalidad», que se realizó mediante análisis de factores y la matriz de correlaciones lineales simples de Pearson. El número de factores implementados resultó igual al número de autovalores iniciales mayores que 1 , y se utilizó la rotación Varimax de Kaiser ${ }^{9}$.

La "confiabilidad o fiabilidad", segunda dimensión de la escala, se evaluó mediante el alfa de Crombach ${ }^{10}$. Este estadístico se acompaña de la determinación de la estructura de asociación de las variables en estudio, mediante el coeficiente de correlación lineal simple de Pearson, y se presenta en un arreglo de valores denominado «matriz de coeficiente de correlación». El análisis de correlación se complementó con uno de conglomerados, el cual consiste en formar grupos homogéneos de variables; para ello se utilizó, como medida de asociación, el valor de la correlación lineal simple y el método jerárquico de la distancia más próxima (obtenido en el acápite anterior). Con el fin de su conformación gráfica, se utilizó el dendograma ${ }^{9}$.

La tercera dimensión de análisis fue la "consistencia de la escala», y para obtenerla se realizó un nuevo análisis de dimensionalidad con una muestra del $50 \%$ de la selección original, de forma aleatoria y por análisis de factores (Mardia, Kent, Bibby, etc.) ${ }^{9}$.

\section{Resultados}

Se incluyeron 219 encuestas. Los resultados de las variables demográficas se muestran en la tabla 1.

Se recibieron encuestas de médicos egresados de 16 diferentes sedes en México; las tres más frecuentes corresponden a escuelas de Ciudad de México (Fig. 1).

En el rubro de satisfacción con la especialidad, del total de los encuestados, 204 (93.2\%) respondieron que se encontraban «muy satisfechos», mientras que $12(5.5 \%)$ contestaron que se hallaban «moderadamente satisfechos" y solo $3(1.4 \%)$ consideraban que se sentían "poco satisfechos". Por orden de frecuencia, los motivos más relevantes fueron que la especialidad en dermatología abarca ámbitos médicos y quirúrgicos, con 101 respuestas (46.1\%); el ámbito académico y de investigación, con 29 (13.2\%); la capacidad de ayudar a los demás, junto con la oportunidad de ver distintos tipos de pacientes con 32 (14.6\%); y tener horarios razonables y tiempo para hacer otras cosas, con 22 (10\%).

En cuanto a la escuela de formación, 165 (75.3\%) de los encuestados respondieron que se encontraban muy satisfechos; 47 (21.5\%) moderadamente satisfechos; 6 (2.7\%) poco satisfechos, y un encuestado no respondió. 
De la población encuestada, 216 individuos (98.6\%) respondieron que volverían a estudiar dermatología como especialidad.

De manera global, en este estudio, al utilizar el IFP, se obtuvo una media de 9.08 ( \pm 1.59$)$; al dividirla en sus dos elementos, se generó en el «bienestar recordado» una media de $9.1( \pm 1.73)$ y en el «bienestar experimentado» una media de $8.78( \pm 1.26)$. De acuerdo con la interpretación de los resultados, se obtuvo un promedio de índice de satisfacción y felicidad «alto», con una media de 9.08 .

La confiabilidad de la escala se determinó con el alfa de Cronbach y resultó un valor de 0.955 , que se interpreta como "alto», ya que este estadístico presenta valores comprendidos en el intervalo de 0 a 1 .

Las variables presentaron valores de asociación positiva y de magnitud de medios a altos, mientras

Tabla 1. Tabla de contingencia demográfica $(n=219)$

\begin{tabular}{llcc}
\hline Sexo & $\mathbf{n}$ & & $\%$ \\
\hline & Mujeres & 159 & 72.6 \\
& Hombres & 60 & 27.4 \\
Edad (años) & Media & 45.68 & 12.20 \\
& Rango & 28 a 79 & \\
Tiempo de ejercicio profesional & Media & 16.17 & 12.23 \\
(años) & & & \\
& Rango & 2 a 50 & \\
\hline
\end{tabular}

que el reactivo «tengo ratos en los que me siento mal» presenta correlaciones nulas o de muy pequeño valor con respecto al resto de las variables en estudio.

El análisis de conglomerados se muestra en el dendograma respectivo y se confirma el grado de asociación de los reactivos de la escala de Pemberton y la disimilaridad de «tengo ratos en los que me siento mal» (Fig. 2).

La dimensionalidad de la escala se estudió mediante un análisis de factores; los dos primeros presentaron una varianza $>1$ y en conjunto representaron un 91.93\% de la varianza total, porcentaje alto para este tipo de análisis. Cada factor se destaca por las variables que presentan un coeficiente alto. Los coeficientes que caracterizan a los factores de las variables de la escala tuvieron un patrón consistente en el análisis de confiabilidad. Los resultados para evaluar la consistencia de la muestra, mediante el análisis de factores en una submuestra del $50 \%$, seleccionada en forma aleatoria, reflejan similares a los del total de la misma $(n=219)$.

\section{Discusión}

La felicidad se define como un estado de grata satisfacción física y espiritual, un estado de ánimo de plenitud y alegría ${ }^{11,12}$. Este concepto está estrechamente relacionado con nuestras percepciones en un

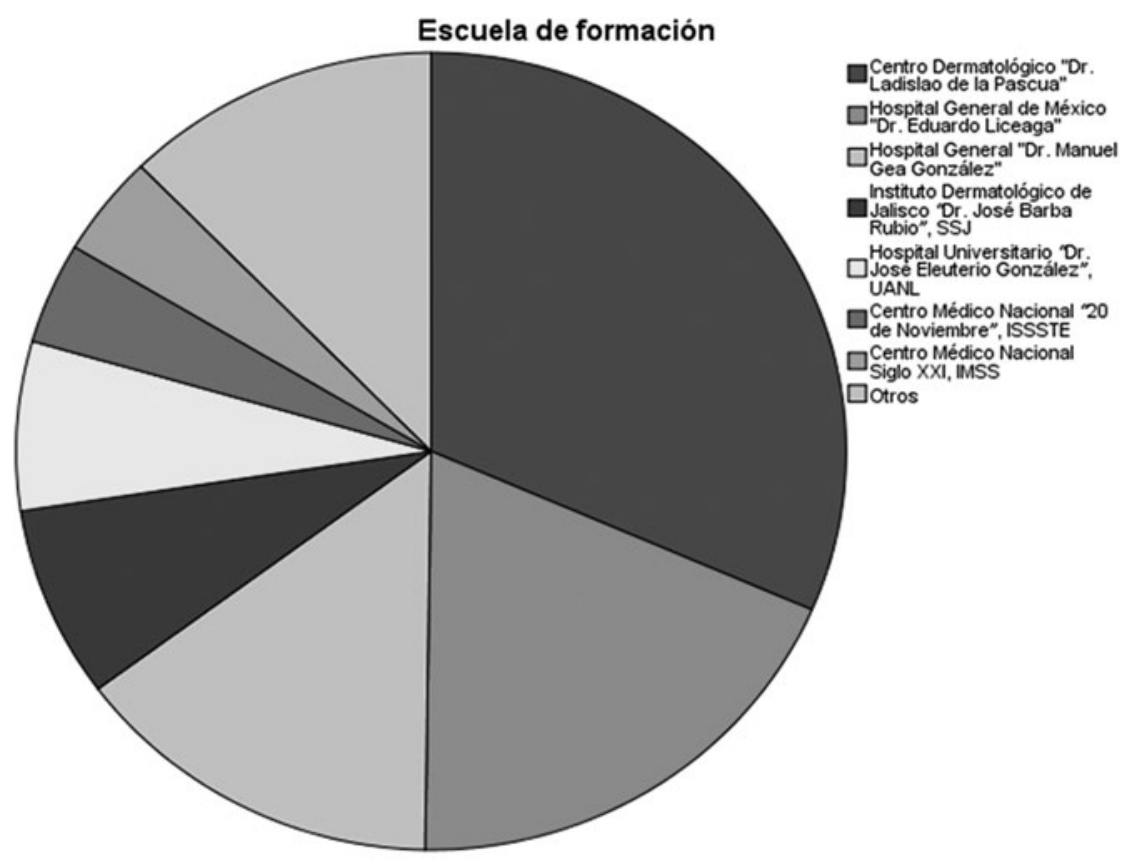

Figura 1. Distribución de la participación de dermatólogos por escuelas. 


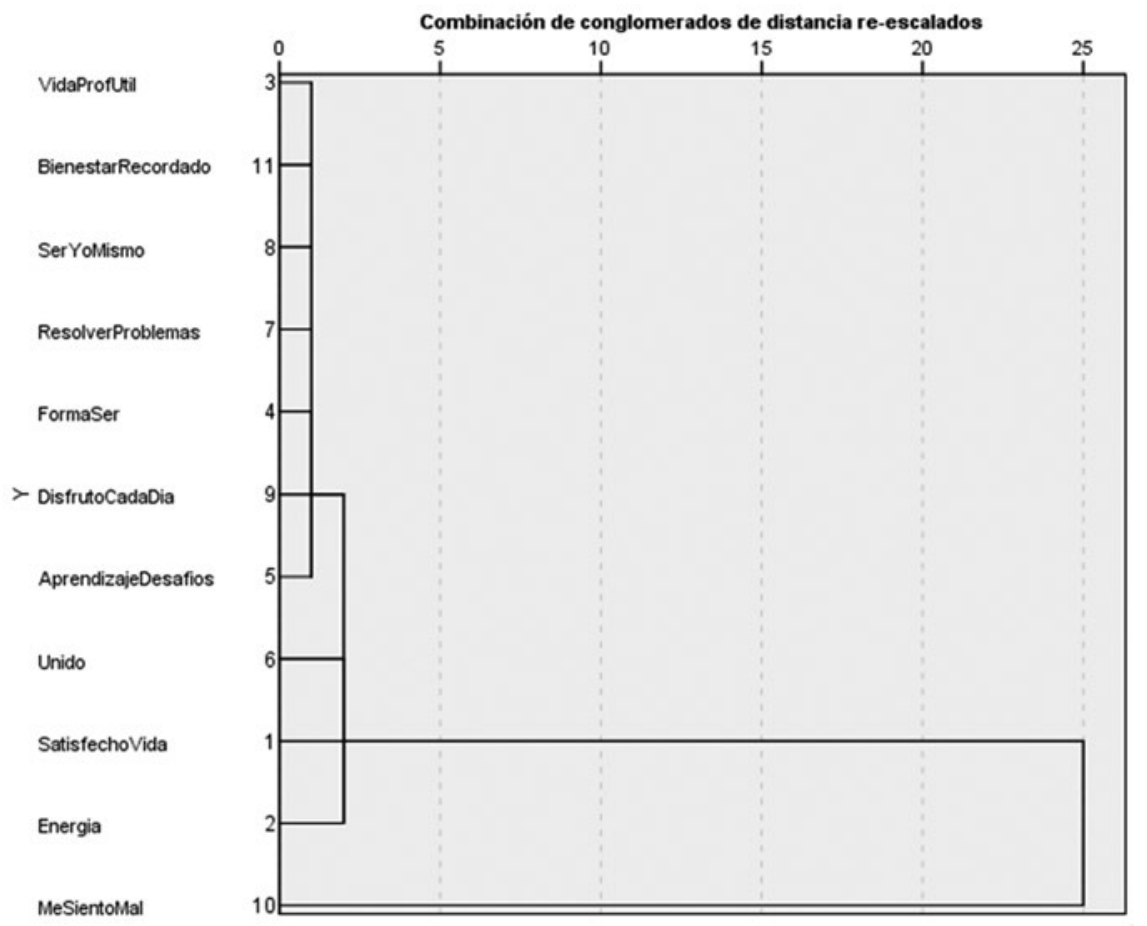

Figura 2. Dendograma de las variables que integran el Índice de Felicidad de Pemberton.

tiempo determinado, por autoevaluación, pero también con la comparación con nuestros semejantes. Se han utilizado las experiencias hedónicas como determinantes de la felicidad, y en la actualidad estas pueden tener relevancia en otros ámbitos de la investigación más allá del área de la salud, por ejemplo económicos e incluso evolutivos, en los que se entiende que la percepción del éxito del individuo depende no solo de sus condiciones económicas, sino también de la actual comparación entre estas condiciones y las previas, la situación de sus pares y las influencias del entorno en que se desenvuelve ${ }^{13}$.

Varios factores de convivencia interpersonal y de estructura en organización laboral se han asociado con el estrés profesional y el desgaste crónico. La percepción de satisfacción laboral tiene un efecto protector contra las consecuencias negativas del estrés en el trabajo. Se ha detallado que, para los profesionistas de la medicina, la satisfacción laboral depende en gran medida de la buena relación con los compañeros (sean superiores o subordinados), de la relación con los pacientes, del tiempo libre disponible, de los valores intrínsecos, del tipo de comunidad donde se realiza la actividad, de la satisfacción con la especialidad, de contar con suficientes recursos, y de su autonomía en el trabajo ${ }^{14,15}$. Varios estudios han reportado que la satisfacción en el trabajo tiene un efecto protector contra el síndrome de desgaste profesional|16,17.
El ejercicio de la medicina ha experimentado cambios relevantes en las últimas décadas, y aunque se han realizado diversos esfuerzos para describir su naturaleza e impacto en la satisfacción de los usuarios, no han permitido entender cómo los médicos experimentan o enfrentan los cambios en la dinámica de su profesión ${ }^{18}$.

La influencia del estrés laboral se ha ampliado y, concretamente en el caso de los profesionales de la salud, es patente y recurrente. Diversos estudios han revelado que, desde la formación de pregrado, los estudiantes tienen mayores niveles de estrés en medicina que en otras carreras y desarrollan diferentes formas de afrontamiento al respecto ${ }^{19}$.

Se han señalado tres elementos sociolaborales vinculados al ámbito profesional del área de la salud que son relevantes en el impacto de la práctica médica y desencadenantes de estrés laboral:

1) Sobrecarga en el número de pacientes que requieren atención, que deriva en una sobrecarga emocional y mental.

2) Aumento en las exigencias macroeconómicas que implican, en consecuencia, un incremento en las necesidades de adaptación de los trabajadores.

3) Deterioro de las condiciones de trabajo con ampliación de jornadas laborales, escasa promoción, cambios en los puestos de trabajo de manera forzada o descendente, cambios de oficio o 
asignaciones, desempleo eventual y jubilación anticipada (no justificada o relacionada con enfermedad ${ }^{20}$.

Se han identificado predictores de la satisfacción laboral, los cuales pueden clasificarse en intrínsecos y extrínsecos. Entre los primeros se encuentran la intención de permanecer en el puesto de trabajo, la posibilidad de afrontar la resolución de problemas, la satisfacción con la vida en general y algunas mediciones de bienestar, como la correlación entre expectativas y logros, el adecuado manejo mental y el apoyo percibido por parte del núcleo familiar. Entre los extrínsecos se encuentran la percepción de apoyo de la organización y las mayores posibilidades de ascenso y promoción en el trabajo21.

Existen diferentes referencias en las que se describe que la satisfacción entre los practicantes de la medicina está en declive ${ }^{22-25}$. El descontento parece explicarse por el aumento de las expectativas o de las exigencias públicas o de los pacientes, por una mayor presión del tiempo laboral, por la restricción en los costos y por los controles regulatorios, que amenazan la autonomía o libertad del ejercicio médico ${ }^{25}$.

La satisfacción profesional es variable en las diferentes especialidades médicas. Esto es de sumo interés e importancia, pues se ha encontrado una fuerte relación entre el bienestar que obtiene el paciente al ser atendido y los resultados que esperaba desde un inicio. La insatisfacción de los médicos en los centros de trabajo origina su necesidad de asociarse en sindicatos, que se manifiesten, que experimenten problemas de salud personales e incluso que dejen por completo la profesión. En síntesis, y para tomarlo en cuenta, la poca satisfacción de los médicos puede aumentar el estrés y los errores en su práctica profesional, lo que pone en riesgo la seguridad de los pacientes.

En años recientes y en medios digitales se han difundido reportes de estudios por encuesta, que no siempre explican las metodologías de investigación, en los cuales los dermatólogos ocupan las primeras posiciones en felicidad; por ejemplo, la empresa de difusión médica Doctoralia ${ }^{\circledR}$ reveló hace algunos meses en medios de prensa digital que en España los dermatólogos se ubican en segundo lugar, detrás de los psicólogos (en México la psicología no es una especialidad médica), y en nuestro país en el primer lugar, seguidos por los pediatras ${ }^{26,27}$.

La fatiga emocional reportada por los dermatólogos es similar a la de los médicos de otras especialidades. Esto puede deberse a que en la relación médico-paciente siempre existirán demandas emocionales, independientemente del área de ejercicio o más allá, por la influencia de los factores organizativos de las instituciones de salud, como que los recursos materiales sean insuficientes o que no se comparta la toma de decisiones, entre otros. Aunque los problemas de índole subjetiva tienen diversos orígenes, los dermatólogos, por ejemplo, tienen que tratar pacientes con enfermedades crónicas que afectan seriamente la calidad de vida, mientras que otros especialistas deben confrontar con mayor frecuencia casos de mutilación o letales ${ }^{28}$.

Las experiencias obtenidas durante la formación de los estudiantes en la escuela de medicina son importantes para determinar su elección de una u otra especialidad $^{29-31}$. Estas incluyen la exposición a un área de trabajo en particular, la convivencia con un líder positivo o las actividades de investigación en un tema específico. Además, hay otros factores que son modificables o no, como la edad, el sexo, el estado marital y las calificaciones en los exámenes. Entre los factores modificables se encuentran el gusto por los retos intelectuales, las oportunidades de futuro para ejercer en la práctica privada, el tiempo de entrenamiento, las condiciones de competencia, la relación a largo plazo con los pacientes, el estilo de vida, si la especialidad implica respeto, la presencia o no de urgencias, el prestigio médico y social, la figura de un modelo a seguir, la recompensa económica, la distribución de género en la especialidad, el reto tecnológico, la diversidad de casos y de pacientes, la experiencia personal con las enfermedades de un área determinada, y la selección de sus compañeros en la carrera ${ }^{32}$.

Dentro de los factores particulares que influyen para que un médico seleccione la dermatología como especialidad están el tiempo que conviven con esta área durante su formación y la experiencia en el ramo antes y después de graduarse, o bien con otras materias relacionadas, como la reumatología o la medicina interna; más allá, uno de los elementos determinantes parece ser la relación que experimente el estudiante con un especialista en la piel, que lo inspire para escoger dicho camino. Algunas otras consideraciones relevantes son la baja competencia, la poca solicitud de urgencias y la posibilidad de tener tiempos compatibles con la vida familiar ${ }^{32,33}$.

Se ha reportado en la literatura internacional que, por diversos motivos, los dermatólogos tienen una mayor satisfacción en el trabajo y menos estrés que los médicos de otras áreas ${ }^{34}$. Es posible entonces que los médicos especialistas en la piel tengan necesidades y percepciones emocionales específicas, las 
cuales establezcan algunas de sus condiciones anímicas, afectivas y de bienestar psicológico, y en consecuencia su satisfacción laboral.

En el año 2007, Clayton, et al. ${ }^{33}$ realizaron en el Reino Unido un estudio con cuestionario electrónico dirigido a miembros de la Sociedad Británica de Dermatología, y concluyeron que el $93 \%$ de los encuestados se encontraba contento de haber elegido la dermatología como especialidad y que pretendían continuar ejerciéndola hasta su retiro. En nuestra investigación tuvimos datos concordantes, pues encontramos que el grado de satisfacción con la especialidad fue del $93.2 \%$.

Los residentes contribuyen a la educación médica otorgando información valiosa durante los ejercicios de retroalimentación, así como en las evaluaciones de los programas académicos del lugar donde se especializan.

En 2008, Freeman, et al. ${ }^{35}$ reportaron un estudio sobre un grupo de residentes de dermatología en los EE.UU., en el que evaluaron el grado de satisfacción con respecto a su entrenamiento y tutoría médica. Concordaron en que la formación tutelar es una influencia positiva, y además relevante en la dirección que toman su carrera y su productividad científica en el área de investigación. El aumento en la satisfacción de su residencia se asoció con las características específicas de los programas, en los que existían tutores de alta calidad y estos tenían tiempo disponible para interactuar con ellos. Concluyeron que es una preocupación constante mantener el interés de los residentes en la práctica clínica y académica con experiencias enriquecedoras por sus tutores, y sugirieron medidas para incrementar el número de aspirantes que busquen una carrera académica. Otro factor que se consideró relevante fue su entrenamiento en las áreas: clínica, de dermatopatología, de dermatología cosmética, de dermatología pediátrica y de gestión empresarial. Cabe señalar que estas tres últimas son poco frecuentes en la mayor parte de los programas de formación y que revelaron generar menor grado de satisfacción.

En nuestros resultados no encontramos relación entre el grado de satisfacción con la especialidad y la escuela de formación, ya que la mayoría se encontró «muy satisfecho» $(75.3 \%)$ o «moderadamente satisfecho" (21.5\%) con su formación académica. Consideramos que esto revela un área de oportunidad para mejorar los programas internos de cada sede hospitalaria y así fomentar el apego al alma mater. La mayor parte de los participantes en la encuesta eran egresados de instituciones médicas de Ciudad de México (64.8\%).

En la actualidad es relevante el conocimiento sobre el bienestar en la vida de las personas; su medición no solo constituye una manera de evaluar los sentimientos y capacidades psicológicas del ser humano, sino también un aspecto central de modelos para la comprensión de la salud psicoemotiva. La evaluación de este fenómeno resulta crucial para validar teorías y modelos de felicidad, así como para medir los resultados de posibles intervenciones favorables, en particular aquellas de índole clínica ${ }^{36}$.

Se han planteado distintos mecanismos de evaluación de la felicidad poblacional y de sus predictores globales. Dentro de ellos se consideran, por ejemplo, el ingreso per cápita, el estado de salud, el soporte social, la libertad de decisión, la generosidad, la percepción de corrupción, los afectos positivos o negativos, las expectativas y más ${ }^{37}$.

Una escala es un constructo diseñado para medir el estado verdadero de una cualidad de interés, y también es una sucesión ordenada de valores distintos pertenecientes a una misma cualidad. Se llama escala a una serie de valores o grados que pueden ubicarse dentro de una misma contingencia o situación cuantitativa $^{38}$. Algunas escalas cubren componentes o aspectos relacionados con el bienestar (satisfacción con la vida, emociones positivas, funcionalidad psicológica, estado social), pero tienen la desventaja de cubrir un solo ámbito. El proceso de análisis emocional es complejo, pues el bienestar psicológico puede evaluarse con diferentes marcadores o con respecto al tiempo (retrospectivo o inmediato), hecho que incrementa la dificultad de desarrollar una medición profunda y comprensiva ${ }^{8}$.

El IFP fue validado en 2013 por Hervás y Vázquez ${ }^{8}$ mediante un análisis de 4052 personas, de entre 16 y 60 años de edad. Identifica factores del bienestar para así otorgar una referencia que abarque los dominios más relevantes indicados en la literatura y en diferentes márgenes de tiempo. Incluye tanto el bienestar que se vive en el momento de su ejecución como el que se recuerda; además, identifica la suma de las experiencias positivas y la ausencia de experiencias negativas del día anterior. Esta herramienta se encuentra validada en siete idiomas (incluyendo el español) y en nueve países (incluyendo México), lo que incrementa el valor transcultural. Por ello decidimos utilizar dicho elemento de análisis para el desarrollo de esta investigación. Nuestros resultados revelan que el IFP es confiable, con una dimensionalidad 
bien definida y además consistente con otros subconjuntos de muestras de la misma población.

En dicha escala obtuvimos una calificación media de 9.08, la cual se interpreta como alta, que podemos definir como un estado de «bienestar profundo" y de "compromiso y satisfacción con la vida»; así, a partir de estas respuestas, el grado de felicidad es alto. Esto coincide con lo descrito en la literatura internacional, que coloca a la dermatología dentro de los primeros lugares de satisfacción en comparación con otras especialidades.

El conocimiento de estas referencias y de las condiciones detectadas en otras latitudes nos permite establecer marcadores de seguimiento y rubros comparativos en los ámbitos emocionales y afectivos en nuestro medio.

Según lo reportado en la literatura estadounidense, la dermatología es una de las especialidades que genera mayor satisfacción entre los médicos, y probablemente esto se deba a: 1) las oportunidades de un empleo próspero; 2) la posibilidad de preservar, por autorreferencia, un constante flujo de pacientes con problemas en la piel; 3) tener horarios de trabajo más aceptables que otras especialidades, y 4) que los resultados del tratamiento frecuentemente son directos, a corto o mediano plazo, y evidentes para los enfermos, lo que favorece la buena relación médico-paciente ${ }^{33,39}$.

En nuestra investigación, los motivos más relevantes de satisfacción fueron elementos relacionados con el ejercicio práctico médico-quirúrgico, tener capacidad de ayudar y el ámbito científico. Además, consideramos que hay otros elementos que podrían influir de manera secundaria o complementaria en la satisfacción revelada, como el estatus social obtenido, la posibilidad de establecer vínculos de amistad, la remuneración económica, el no enfrentar casos letales con frecuencia, el no tener guardias tan demandantes o frecuentes como en otras especialidades, el viajar frecuentemente a congresos médicos, y el tener horarios razonables y en consecuencia tiempo disponible para realizar otras actividades además del trabajo, entre otros.

El estudio de Arias-Santiago, et al.$^{40}$, realizado en Granada, España, en el año 2011, reportó que la calidad de vida en un grupo de dermatólogos locales fue moderada, pues aunque tenían una fuerte motivación intrínseca, el apoyo que recibían de sus superiores era de bajo a moderado, y tenían gran cantidad de trabajo y alto grado de responsabilidad. Interpretamos que los resultados difieren de los nuestros debido a que el sistema de salud en España es distinto al mexicano; sin embargo, sería interesante más adelante evaluar las diferencias de satisfacción entre los médicos que trabajan en una institución y los que ejercen solo la medicina privada o los que realizan ambas prácticas, tanto en un país como en otro.

El más actual y completo estudio sobre los estados de satisfacción en las compensaciones de los médicos fue realizado por Peckham ${ }^{41}$ en 2016, con una encuesta a más de 19,000 médicos de 26 especialidades en los EE.UU. Para ellos, los principales aspectos de recompensa laboral fueron la gratitud y la relación con los pacientes. Los datos relevantes para los dermatólogos indicaron que son los terceros con mayores percepciones económicas al año, por detrás de los ortopedistas y los cardiólogos; y son número uno en sentirse justamente gratificados, satisfechos con su carrera y especialidad, y en consecuencia con su profesión integral. Nos llama la atención que solo el $74 \%$ de los encuestados volvería a escoger esta especialidad, mientras que en nuestro reporte se alcanzaron cifras cercanas al $99 \%$.

Al evaluar los factores de la dimensionalidad, los mayores valores se encontraron en los reactivos "Creo que mi vida profesional es útil y valiosa» y "creo que, en lo importante, puedo ser yo mismo", situaciones que pueden traducirse en que el hecho de sentirnos útiles en la profesión y la sensación de autonomía son factores relevantes.

Aunque al momento no hay estudios locales al respecto, nos cuestionamos si las influencias particulares de nuestro medio y entorno, como pueden ser étnicas, sociales, culturales, económicas, de participación en la comunidad, de jerarquización pública, adquisitivas y otras, podrían ser estímulos de percepción de felicidad en la generalidad médica. Los estudios más amplios y recientes sobre felicidad en todo el mundo ubican a México en la posición 21 del tabulador global y en cuarto lugar en América Latina, después de Costa Rica, Puerto Rico y Brasil ${ }^{37}$.

Otros elementos podrían ser el tiempo que se distribuye entre la práctica institucional y privada, o las horas dedicadas específicamente a la jornada laboral y la retribución en dinero al respecto. En México no se ha identificado el nivel de percepción monetaria en las diferentes especialidades, mientras que en los EE.UU., como ya mencionamos, los dermatólogos ocupan el tercer lugar en ese rubro ${ }^{41}$.

Estimamos que los datos vertidos en este tipo de investigaciones, entre otras cosas, revelarán la posibilidad de instaurar herramientas para confrontar fenómenos en el ejercicio del médico, como es el síndrome de desgaste profesional (en la Clasificación 
Internacional de Enfermedades, CIE-z73.0), en el que hay altos grados de agotamiento emocional, despersonalización y baja realización propia ${ }^{42,43}$.

Un reporte sobre el estilo de vida en los médicos de los EE.UU. ${ }^{44}$ indicó que los dermatólogos tienen bajos niveles de síndrome de desgaste profesional en comparación con otros especialistas y que constituyen el número uno en estado de felicidad, tanto dentro como fuera del trabajo; además, son los que más ejercicio hacen a la semana y los que tienen menores índices de sobrepeso.

Un dato interesante es la distribución por sexo, pues más del $70 \%$ de los participantes en nuestro estudio fueron mujeres y también en otros lugares se ha reportado que la dermatología es una de las especialidades en las que ellas predominan, tras la ginecología y la obstetricia, la pediatría, la patología y la psiquiatría ${ }^{41}$. ¿Será entonces que las particulares percepciones de la felicidad femeninas influyen en las estadísticas? Creemos que sí.

Entendemos que las características singulares del entorno en que se desarrollan los dermatólogos en México son favorables, no solo en el ámbito profesional, sino que también se acompañan de elementos de bienestar social, económico, familiar, climatológico y de convivencia interpersonal, que en nuestro ámbito cultural se destacan como de gran aprecio y valor para la vida misma.

\section{Conclusiones}

Podemos decir que los dermatólogos en México tienen altos índices de satisfacción y felicidad en cuanto a su vida y profesión.

Al momento del estudio, en nuestro país había 16 sedes hospitalarias dedicadas a la formación académica de especialistas en dermatología, y la mayoría de los participantes se encontraron muy satisfechos con su escuela de formación en posgrado.

Los motivos más relevantes para manifestarse positivos con la especialidad fueron relacionados con las esferas del ejercicio médico y humanista. Percibimos que la dermatología permite tener satisfacciones profesionales en distintos terrenos, lo que es compatible con la posibilidad de obtener una realización en la vida personal y familiar del especialista, y de ahí que casi la totalidad de los encuestados volvería a escoger esta rama de la medicina.

Entre los dermatólogos es de suma importancia el hecho de sentirse útiles, valiosos y con la capacidad de mantener autonomía.
El uso del IFP funcionó muy bien como escala de evaluación en nuestra investigación.

Hasta donde conocemos, este es el primer estudio al respecto en nuestro medio, y además de poder despertar interés en el ámbito académico, consideramos que servirá de precedente para nuevas investigaciones.

El presente trabajo contó con la aprobación y los registros correspondientes en la Dirección de Investigación (DI/15/109/03/086), el Comité de Investigación (Cl/88/15) y el Comité de Ética (CE/737/15) del Hospital General de México Dr. Eduardo Liceaga, de la Secretaría de Salud.

\section{Agradecimientos}

Agradecemos a la Sociedad Mexicana de Dermatología, a la Academia Mexicana de Dermatología y a la Sociedad Mexicana de Cirugía Dermatológica y Oncológica, por el apoyo en la difusión del instrumento de encuesta. A la Dra. Julieta Fierro-Gossman, por la asesoría conceptual en este proyecto.

\section{Bibliografía}

1. McMahon DM. Happiness: a history. New York: Grove Press; 2006. p. 353-453.

2. Organización Mundial de la Salud. Salud mental: un estado de bienestar. Diciembre 2013. (Consultado en junio de 2016.) Disponible en: www. who.int/features/factfiles/mental health/es

3. Vázquez C, Hervás G, Rahona JJ, et al. Bienestar psicológico y salud: aportaciones desde la psicología positiva. Ann Clin Health Psychol. 2009;5:15-28.

4. Ryff CD. Psychological well-being in adult life. Curr Dir Psychol Sci. 1995;4:99-104.

5. Vázquez C, Duque A, Hervás G. Satisfaction with life scale in a representative sample of Spanish adults: validation and normative data. Span J Psychol. 2013;16:1-15

6. Warr P. Sources of happiness and unhappiness in the workplace: a combined perspective. J Work Organizational Psychol. 2013;29:99-106.

7. Daniel WW. Bioestadística: base para el análisis de las ciencias de la salud. $4^{a}$ ed. México: Limusa Wiley; 2001. p. 138.

8. Hervás $\mathrm{G}$, Vázquez $\mathrm{C}$. Construction and validation of a measure of integrative well-being in seven languages: the Pemberton Happiness Index. Health Qual Life Outcomes. 2013;11:66.

9. Hair JF Jr, Black WC, Babin BJ, Anderson RE. Multivariate data analisis. $7^{\text {th }}$ ed. New Jersey: Prentice Hall; 2010. p. 91-138, 469-518.

10. Netemeyer RG, Bearden WO, Sharma S. Scaling procedures: issues and applications. California: Sage; 2003. p. 8-15.

11. Real Academia Española. Diccionario de la Lengua Española. (Consultado en junio de 2016.) Disponible en: http://dle.rae.es/?id=Hj4JtKk

12. Academia Mexicana de la Lengua. (Consultado en junio de 2016.) Disponible en: http://www.academia.org.mx/felicidad

13. Rayo L, Becker GS. Evolutionary efficiency and happiness. Journal of Political Economy. 2007;115:302-37.

14. Williams ES, Konrad TR, Linzer M, et al. Refining the measurement of physician job satisfaction: results from the Physician Worklife Survey. SGIM Career Satisfaction Study Group. Society of General Internal Medicine. Med Care. 1999;37:1140-54.

15. Konrad TR, Williams ES, Linzer M. Measuring physician job satisfaction in a changing workplace and a challenging environment. SGIM Career Satisfaction Study Group. Society of General Internal Medicine. Med Care. 1999;37:1174-82.

16. Aiken LH, Clarke SP, Sloane DM, et al. Hospital nurse staffing and patient mortality, nurse burnout, and job dissatisfaction. JAMA. 2002; 288:1987-93.

17. Ramírez AJ, Graham J, Richards MA, et al Mental health of hospital consultants: the effects of stress and satisfaction at work. Lancet. $1996 ; 347: 724-8$. 
18. Bascuñán ML. Cambios en la relación médico-paciente y nivel de satisfacción de los médicos. Rev Med Chile. 2005;133:11-6.

19. De la Rosa-Rojas G, Chang-Grozo S, Delgado-Flores L, et al. Niveles de estrés y formas de afrontamiento en estudiantes de Medicina en comparación con estudiantes de otras escuelas. Gac Med Mex. 2015;151:443-9.

20. Mingote-Adán JC, Moreno-Jiménez B, Gálvez-Herrer M. Burnout and the health of the medical professionals: review and preventive offers. Med Clin (Barc). 2004;123:265-70.

21. Paris L, Omar A. Predictors of job satisfaction among physicians and nurses. Estudios de Psicología. 2008;13:233-44.

22. Kassirer J. Doctor discontent. N Engl J Med. 1998;339:1543-5.

23. Smith R. Why are doctors so unhappy? There are probably many causes, some of them deep. BMJ. 2001;322:1073-4.

24. Zuger A. Dissatisfaction with medical practice. N Eng J Med. 2004;350:69-75

25. Nylenna M, Glubrandsen P, Førde R, et al. Job satisfaction among Norwegian general practitioners. Scand J Prim Health Care. 2005; 23:198-202.

26. IM Médico. El ranking de médicos más felices de España según su especialidad. (Consultado en junio de 2016.) Disponible en: http://www. immedicohospitalario.es/noticia/7217/el-ranking-de-medicos-mas-felices-de-espana-segun-su-especialidad

27. Notimex. ¿Quiénes son los profesionales más felices? Disponible en http://www.zocalo.com.mx/seccion/articulo/quienes-son-los-profesionales-mas-felices-1458309442

28. Renzi C, Tabolli S, lanni A, et al. Burnout and job satisfaction comparing healthcare staff of a dermatological hospital and a general hospital. J Eur Acad Dermatol Venereol. 2005;19:154-7.

29. Schwartz RW, Jarecky RK, Strodel WE, et al. Controllable lifestyle: a new factor in career choice by medical students. Acad Med. 1989;64:606-9.

30. Erzurum VZ, Obermeyer RJ, Fecher A, et al. What influences medical students' choice of surgical careers. Surgery. 2000;128:253-6.

31. Chen $\mathrm{H}$, Hardacre JM, Martin C, et al. Do medical school surgical rotations influence subspecialty choice? J Surg Res. 2001;97:172-8.
32. Ibrahim M, Fanshawe A, Patel V, et al. What factors influence British medical students' career intentions? Med Teach. 2014;36:1067-72.

33. Clayton R, Lambert T, Burge SM. Career choices in dermatology. Clin Exp Dermatol. 2010;35:86-90.

34. Leigh JP, Tancredi DJ, Kravitz RL. Physician career satisfaction within specialties. BMC Health Serv Res. 2009;9:166.

35. Freeman SR, Greene RE, Kimball AB, et al. US dematology residents' satisfaction with training and mentoring: survey results from the 2005 and 2006 Las Vegas Dermatology Seminars. Arch Dermatol. 2008; 144:896-900.

36. Diener E. Subjective well-being. The science of happiness and a proposal for a national index. Am Psychol. 2000:55:34-43.

37. Helliwell J, Layard R, Sachs J. World Happiness Report 2016, Update (Vol. I). New York: Sustainable Development Solutions Network. 2016. (Consultado en junio de 2016.) Disponible en: http://worldhappiness.report/wp-content/uploads/sites/2/2016/03/HR-V1_web.pdf

38. DefiniciónABC. Escala. (Consultado en junio de 2016.) Disponible en: http://www.definicionabc.com/ciencia/escala.php

39. Kimball AB. Dermatology: a unique case of specialty work-force economics. J Am Acad Dermatol. 2003;48:265-70.

40. Arias-Santiago S, Buendía-Elsman A, Husein-ElAhmed H, et al. Perception of dermatologist quality of life. J Am Acad Dermatol. 2011; 64:S1-AB80.

41. Peckham C. Medscape physician compensation report. Medscape apr. 1, 2016. (Consultado en junio de 2016.) Disponible en: http://www.medscape.com/features/slideshow/compensation/2016/public/overview

42. Hidalgo-Vicario MI. Burnout syndrome. Ped Int. 2016;20:5-6.

43. Catálogo de Enfermedades eCIE10ES, Gobierno de España. Ministerio de Sanidad, Servicios Sociales e Igualdad. Disponible en: http://eciemaps.msssi.gob.es/ecieMaps/browser/indexMapping.html\#code $=Z 73.0 \&$ source $=$ cie10 $\mathrm{mc} \&$ target $=c i e 9 \mathrm{mc}$

44. Peckerham C. Medscape lifestyle report 2016: bias and burnout. Medscape Jan 13, 2016. Disponible en: https://www.medscape.com/slideshow/lifestyle-2016-overview-6007335\#page=1 